\title{
Comprometimento organizacional atitudinal: um estudo empírico sobre a dimensionalidade do construto
}

\author{
Attitudinal organizational commitment: an empirical \\ research study into the construct dimensionality
}

\author{
Igor Gomes MENEZES \\ Antonio Virgílio Bittencourt BASTOS
}

\begin{abstract}
Resumo
A falta de precisão acerca da dimensionalidade do comprometimento organizacional contribui para torná-lo um construto complexo e multifacetado. Com o objetivo de identificar a melhor estrutura fatorial para o comprometimento organizacional, o presente trabalhou testou o inter-relacionamento de duas medidas atitudinais - afetiva e instrumental - bem como o relacionamento dessas variáveis com uma medida de intenções comportamentais. Foram selecionados 1869 trabalhadores das regiões Norte, Nordeste e Sul do Brasil. Os resultados das análises de regressão e das análises fatoriais exploratórias e confirmatórias apontaram a bidimensionalidade da base instrumental (falta de alternativas e sacrifícios percebidos). Por outro lado, demonstraram que a dimensão instrumental não compõe a estrutura fatorial do comprometimento organizacional atitudinal que, por ora, é um construto unidimensional, formado somente pela dimensão afetiva.
\end{abstract}

Unitermos: Atitude profissional. Comprometimento organizacional. Dimensionalidade do construto. Perspectiva comportamental.

\begin{abstract}
The lack of precision surrounding the dimensionality of organizational commitment helps to make it a complex and multifaceted construct. In order to identify the best factor structure for organizational commitment, this study tested the inter-relationship between two attitudinal measures (affective and instrumental) and also the relationship of these variables with a measurement of behavioral intentions. We selected 1,869 workers from the Northern, Northeastern and Southern regions of Brazil. The results of the regression analysis and both the exploratory and confirmatory factor analysis showed the bidimensionality of the instrumental dimension (lack of alternatives and high sacrifices); on the other hand, they demonstrated that this dimension is not part of the factor structure of attitudinal organizational commitment, which in turn is a one dimensional construct, comprising only the affective dimension.
\end{abstract}

Uniterms: Occupational attitudes. Organizational commitment. Construct dimensionality. Behavior.

Uma das maiores preocupações teóricas e empíricas que cercam as pesquisas sobre comprometimento organizacional, dentro da perspectiva atitudinal, diz respeito ao estudo da sua dimensiona- lidade. Não existe um consenso na literatura acerca de quantas e quais seriam as dimensões constitutivas desse tipo de vínculo estabelecido entre trabalhador e organização, o que faz do comprometimento

$\cot$

- Universidade Federal da Bahia, Centro de Estudos Interdisciplinares para o Setor Público. Av. Ademar de Barros, s/n., Pavilhão 4, Campus Universitário de Ondina, 40170-110, Salvador, BA, Brasil. Correspondência para/Correspondence to: I.G. MENEZES. E-mail: <igorgmenezes@gmail.com>. 
organizacional um construto complexo e multifacetado.

Autores como Mowday, Porter e Steers (1982) consideram o comprometimento como um conceito unidimensional, que tem como base o sentimento de identificação. Meyer e Allen (1984) compreendem o comprometimento a partir de duas bases: a afetiva, que corresponde a gostar ou identificar-se com a organização, e a instrumental, que consiste no interesse de manter-se vinculado à organização por necessidades. Em 1990, os mesmo autores já concebem o comprometimento como um construto tridimensional, adicionando a base normativa, relacionada ao sentimento de dever e obrigação, como justificativa para o comprometimento juntamente com as demais bases.

Após o desenvolvimento da perspectiva tridimensional e com base nos estudos sobre a dimensionalidade do Organizational Commitment Questionnaire (OCQ), de Bar-Hayim e Berman (1992), Mowday, Steers e Porter (1979) apostam na existência de unicamente dois tipos de comprometimento: um comprometimento passivo, ligado às noções de lealdade e desejo de permanecer; e um comprometimento ativo, traduzido na identificação e envolvimento do indivíduo. Delobbe e Vandenberghe (2000) investigam o comprometimento mediante o estudo de quatro dimensões: internalização, afetiva, de continuação e aquiescência. No Brasil, Medeiros, Albuquerque, Marques e Siqueira (2005) hipotetizaram sete bases para o comprometimento organizacional: afetiva, obrigação em permanecer, obrigação pelo desempenho, afiliativa, falta de recompensas e oportunidade, linha consistente de atividade e escassez de alternativas. Recentemente, Solinger, van Ollfen e Roe (2008) conduziram uma pesquisa em que foi identificado um fator como o mais característico: o afetivo, fortemente correlacionado com a dimensão normativa.

Tendo em vista, assim, a multiplicidade de interpretações sobre a composição teórica do construto, são três as principais discussões que permeiam os estudos sobre a dimensionalidade do comprometimento organizacional: 10) a possibilidade de que haja uma sobreposição conceitual entre as bases afetiva e normativa (Bastos, 1994; Cohen, 2003; Hartmann \& Bambacas, 2000; Ko, Price \& Mueller, 1997; Lee \& Chulguen, 2005; Mathieu \& Zajac, 1990; McGee \& Ford, 464 1987; Menezes, 2006; Meyer, Stanley, Herscovitch \&
Topolnytsky, 2002; Shore, Tetrick, Shore \& Barksdale, 2000; Solinger et al., 2008); 20) a factibilidade de a base instrumental apresentar duas subdimensões distintas (Allen \& Meyer, 1996; Hackett, Bycio \& Hausdorf, 1994; Hartmann \& Bambacas, 2000; Labatmediene, Endriulaitiene \& Gustainiene, 2007; Mathieu \& Zajac, 1990; McGee \& Ford, 1987; Meyer \& Allen, 1991; Meyer, Allen \& Gellatly, 1990; Solinger et al., 2008), mas não constitutivas do comprometimento organizacional (Lee \& Chulguen, 2005; Meyer et al., 2002; Solinger et al., 2008); e 30) a possibilidade de que o comprometimento organizacional seja um construto unidimensional, formado unicamente pela base afetiva (Mowday et al., 1982; Solinger et al., 2008).

Em face dos elementos discutidos acima, o presente estudo tem como propósito testar a dimensionalidade do comprometimento organizacional, investigando a inter-relação entre diferentes variáveis atitudinais.

\section{A dimensionalidade do comprometimento organizacional}

Dentre as discussões atuais sobre a dimensionalidade do comprometimento organizacional, a que tem revelado maior consenso entre os resultados de pesquisas empíricas é a sobreposição conceitual entre as bases afetiva e normativa desse construto. Desde o clássico estudo de validação do modelo tripartite de comprometimento organizacional, de Meyer, Allen e Smith (1993), verificou-se que as dimensões afetiva e normativa apresentavam sobreposição conceitual, quando avaliadas a partir da Affective Commitment Scale (ACS) e da Normative Commitment Scale (NCS).

A hipótese central para a ocorrência dessa sobreposição reside no fato de que o sentimento de dever e obrigação, proveniente da internalização das normas, advém geralmente de um processo prévio de identificação com a organização, ou seja, do vínculo afetivo que o trabalhador mantém com a organização. Essa hipótese encontra-se sustentada nos pressupostos teóricos da teoria da influência social, de Kelman (1958), para quem a dimensão normativa (internalização) encontra-se correlacionada tanto com a natureza afetiva quanto com a base instrumental, sendo essas duas últimas dimensões as mais significativas para representar 
o tipo de vínculo estabelecido entre o indivíduo e o grupo ao qual ele se mantém afiliado.

A segunda discussão em torno da dimensionalidade do comprometimento organizacional envolve a dimensão mais controversa e que tem promovido continuamente novas reflexões sobre a operacionalização do construto: a dimensão instrumental, denominada também calculativa ou de continuação. Uma pesquisa conduzida por McGee \& Ford (1987) para testar o modelo bidimensional de Meyer e Allen (1984), verificou que a melhor solução fatorial para a Continuance Commitment Scale (CCS) não envolvia um fator, mas dois. Enquanto a ACS mostrou-se bastante coesa, a CCS aparentou avaliar duas subdimensões: $1^{\text {a) }}$ falta de alternativas ou oportunidades de trabalho (CC:LoAlt); e 2a) sacrifícios percebidos ao deixar a organização (CC:HiSac).

Os resultados das pesquisas que apontaram a bidimensionalidade da CCS acabaram por suscitar uma nova questão. Se a dimensão instrumental não possui uma estrutura fatorial homogênea, pode-se aceitá-la como uma dimensão constitutiva do comprometimento organizacional? Tendo por base essa reflexão, alguns estudos foram conduzidos a fim de testar o relacionamento entre as duas subdimensões (CC:LoAlt e CC:HiSac) com a ACS e a NCS. No estudo de McGee \& Ford (1987), foi encontrada uma correlação significativamente negativa entre a ACS e CC:LoAlt, enquanto, por outro lado, os níveis de correlação entre ACS e CC:HiSac foram significativamente positivos. Os resultados da meta-análise realizada por Meyer et al. (2002) demonstraram que o mesmo padrão de correlação da ACS com CC:LoAlt e CC:HiSac tem ocorrido entre a NCS e as subdimensões daCCS.

A falta de clareza conceitual da dimensão instrumental não reside unicamente na divisão em duas subdimensões. A base instrumental está diretamente relacionada à noção de permanência na organização e apoia-se na teoria dos side-bets de Becker (1960). Esta, por sua vez, é consistente com a teoria da troca, segundo a qual o trabalhador se sente recompensado com o que lhe é oferecido pela organização, e percebe que sua saída implicaria sacrifícios econômicos, sociais e/ou psicológicos.

A associação entre a dimensão instrumental e a permanência na organização gera um problema adicional ao estudo da constituição dimensional do cons- truto, visto que associa o que deveria ser o fator justificador do vínculo de comprometimento (que seria a base instrumental) a um consequente do comprometimento (que seria o desejo de permanecer como membro da organização, em face dos possíveis custos ligados a sua saída). Se assim se procede, o vínculo instrumental serviria muito mais como um preditor do desejo de permanência na organização do que como um constituinte do comprometimento organizacional, já que não se pode garantir que um trabalhador venha a assumir uma postura proativa de comprometimento somente por acreditar que sair da organização lhe acarretaria prejuízos.

Resultados da pesquisa conduzida por Randall, Cropanzano, Bormann e Biriulin (1997) destacaram que o comportamento efetivo de deixar a organização pode ser predito pelo comprometimento de continuação. Além dessa pesquisa, de acordo Hartmann e Bambacas (2000), a intenção de saída da organização encontrou-se fortemente associada a CC:LoAlt $(r=0,77, p<0,01)$, corroborando o trabalho clássico de Arnold e Feldman (1982), que encontraram uma correlação de $r=0,44$ $(p<0,01)$ entre intenção de turnover e intenção pela procura de outras alternativas de trabalho. Da mesma forma, CC:HiSac correlacionou-se positivamente com as intenções de permanência $(r=0,42, p<0,01)$.

Tendo em vista assim (a) a forte associação entre o comprometimento de continuação e a noção de permanência na organização; (b) os resultados de correlação negativa entre CC:LoAlt e ACS; (c) a falta de precisão em relação à subdimensão CC:HiSac, que se mostra positivamente correlacionada tanto com a CC:LoAlt quanto com a ACS; e (d) o forte grau de correlação entre a ACS e a NCS, há uma tendência atual em se reconhecer a base afetiva como o único componente constituinte do comprometimento organizacional atitudinal.

Adicionalmente, estudos mais recentes têm demonstrado que a dimensão instrumental não possui correlações satisfatórias com medidas gerais de comprometimento, como o OCQ, o que lança dúvida sobre a validade convergente da base instrumental (Lee \& Chulguen, 2005; Meyer et al., 2002; Solinger et al., 2008).

À luz desse conjunto de achados de pesquisa, o presente trabalho assume, a princípio, o pressuposto de que a dimensão afetiva do comprometimento organi- 
zacional sobrepõe-se conceitualmente à base normativa. Assim, foram elaboradas quatro hipóteses para testar a relação entre as perspectivas afetiva e instrumental:

$\mathrm{H}_{1}$ : a dimensão instrumental do comprometimento organizacional é composta por duas subdimensões (falta de alternativas ou oportunidades de trabalho e sacrifícios percebidos ao deixar a organização);

$\mathrm{H}_{2}$ : a dimensão instrumental não compõe a estrutura fatorial do comprometimento organizacional atitudinal;

$\mathrm{H}_{3}$ : o comprometimento organizacional é um construto unidimensional, formado unicamente pela dimensão afetiva;

$\mathrm{H}_{4}$ : a dimensão afetiva é a única a apresentar poder preditivo sobre as intenções comportamentais de comprometimento organizacional.

\section{Método}

\section{Participantes}

Para a testagem das hipóteses de pesquisa foram selecionados, por amostragem por julgamento, 1989 trabalhadores de empresas do setor primário $(27,7 \%)$, secundário $(44,6 \%)$ e terciário $(27,7 \%)$ da economia, situadas no Polo Industrial de Manaus, na Região do Baixo Médio São Francisco (Juazeiro e Petrolina) e na Região Metropolitana de Florianópolis. Apresentando características culturais distintas, as três regiões foram escolhidas com o intuito de aumentar a variabilidade da amostra e aumentar o poder de generalização dos resultados da investigação. Dos 1989 casos iniciais, 120 foram excluídos do banco de dados final por atender a pelo menos um dos seguintes critérios: $1^{\circ}$ ) ter mais de 10,0\% de respostas missing (Troyanskaya et al., 2001; Wagner, Motta \& Dornelles, 2004); 20) possuir um valor de soma superior a 12 na Escala de Validade; e $3^{\circ}$ ) estar distante a mais de 1,5 da amplitude interquartílica, sendo considerado um caso outlier.

Dos 1869 casos válidos, 1025 (55,0\%) eram do gênero masculino, sendo a média de idade de 31,25 anos (Desvio-Padrão - DP=10,27). Dentre os participantes, 42,6\% possuíam nível médio completo; 26,9\%, nível superior incompleto; 18,6\%; nível superior completo; e
11,9\% eram pós-graduados. Em relação às organizações investigadas, 20,7\% possuíam menos de 50 trabalhadores; 7,1\%, de 50 a 99 trabalhadores; 18,6\%, de 100 a 249 trabalhadores; 14,5\%, de 250 a 499 trabalhadores; e 39,1\%, acima de 500 trabalhadores. Quanto ao empregador, 77,1\% eram empresas privadas; 18,1\%, órgãos públicos; e 3,9\%, outros tipos de organização (ONG e cooperativas).

\section{Instrumentos}

Para o presente estudo, o questionário de pesquisa foi composto por três escalas, sendo duas escalas utilizadas para avaliação de características atitudinais do comprometimento organizacional, e uma escala utilizada para a predição das intenções comportamentais em face das medidas atitudinais.

A primeira das escalas atitudinais adotadas buscou adaptar alguns itens do OCQ e da ACS, para a composição de uma medida geral de comprometimento afetivo. Esta estratégia fora adotada no Brasil por Bastos (1994) e Medeiros (2003). Foram adaptados, assim, novamente 7 dos 15 itens da versão original do OCQ, outrora traduzida e adaptada para o contexto nacional por Borges-Andrade, Afanasieffe Silva (1989), e 3 dos 6 itens da ACS, traduzida e adaptada para o país por Medeiros e Enders (1998) e, agora, readaptada para a presente pesquisa. A readaptação dos itens teve como objetivo selecionar descritores mais precisos e diretos para a avaliação do comprometimento organizacional, além de torná-los de mais fácil compreensão para os respondentes. A junção do OCQ com a ACS - denominada aqui como AFET - buscou atender à finalidade de avaliar em uma medida única o comprometimento afetivo, a partir de um total de 10 itens.

Para a avaliação da subdimensão "falta de alternativas ou oportunidades de trabalho" (CC:LoAlt) e "sacrifícios percebidos ao deixar a organização" (CC:HiSac) foram adaptados alguns itens de diferentes instrumentos sobre comprometimento (Carson, Carson \& Bedeian, 1995, Meyer et al., 1993; Powell \& Meyer, 2004; Rego, 2003), a fim de melhorar a sua qualidade psicométrica geral. A escala construída buscou contemplar as características tanto da CC:LoAlt quanto da CC:HiSac. Para a avaliação da CC:LoAlt, foram desenvolvidos dois novos itens; e, para a mensuração da CC:HiSac, cinco novo itens, não disponíveis nas escalas já validadas. As 
medidas atitudinais utilizaram-se da técnica de escalonamento do tipo Likert, com sete categorias de resposta, variando do extremo nível de concordância ao grau máximo de discordância.

Para verificar se as escalas atitudinais apresentavam poder preditivo sobre as intenções comportamentais de comprometimento organizacional, foi aplicada a Escala de Intenções Comportamentais de Comprometimento Organizacional (EICCO), validada por Menezes (2006). A ElCCO foi um instrumento desenvolvido com o objetivo de avaliar os descritores comportamentais associados a posturas que demonstrassem comprometimento organizacional, podendo ser considerada uma escala que integra as perspectivas atitudinal e comportamental em uma medida única.

A técnica de escalonamento adotada foi a de diferencial semântico, com sete intervalos de resposta. Adicionalmente aos itens dessas escalas foram investigadas características pessoais e profissionais, tais como gênero, idade, estado civil, tempo de serviço, tamanho da empresa, dentre outras.

\section{Procedimentos}

Os dados foram coletados nas organizações no momento do expediente, sendo a aplicação do questionário acompanhada pelo pesquisador ou por um bolsista de iniciação científica, previamente treinado. Além dos casos de aplicação da versão impressa das escalas, a coleta de dados ocorreu também mediante o emprego de bancos de dados on-line e off-line, que guardam a mesma configuração e disposição gráfica da versão impressa.

O estudo foi aprovado pelo Comitê de Ética em Pesquisa da Universidade Federal do Amazonas, sob protocolo $n^{\circ}$ CAAE 0013.0.115.000-08, em 28 de março de 2008, tendo todos os participantes assinado o Termo de Consentimento Livre e Esclarecido antes de sua inclusão na amostra.

Para o estudo da validade de construto das medidas atitudinais, foram empregadas diferentes técnicas psicométricas da TCT e da TRI. Pela TCT, o primeiro procedimento adotado foi a realização de uma análise fatorial exploratória, com todos os itens da AFET e das subdimensões CC:LoAlt e CC:HiSac, a fim de verificar qual estrutura fatorial responde pela melhor variância explicada do construto. Pela TRI, as medidas atitudinais foram submetidas à Análise Fatorial por correlações policóricas, visando testar a possibilidade de cada uma das medidas assumir uma estrutura fatorial unidimensional.

Posteriormente, foi realizada a análise de itens, considerando o Rating Scale Model, modelo de análise de Rasch, que consiste em uma implementação da TRI para itens de escala graduada. Tal modelo possibilitou a realização da análise de resíduos das escalas, com o intuito de verificar padrões de resposta não esperados para os itens. Nessa análise, os parâmetros avaliados foram infit mean square e outfitmean square. O infit reflete a falta de ajuste entre as respostas de sujeitos com elevado comprometimento em itens em que se esperaria que ele respondesse de forma comprometida (erro inesperado), e as respostas de alto comprometimento por parte de sujeitos com baixo comprometimento (acerto inesperado). Já o outfit aponta o sujeito que não adere a itens muito fáceis para seu nível, ou que acerta itens muito distantes do seu nível. Para amostras maiores ( $N>1000)$, os valores de infit e outfit devem situar-se entre 0,80 e 1,20 (Bond \& Fox, 2007). Uma vez garantida a validade de construto pela TCT e pela TRI, procedeu-se ao exame da fidedignidade das medidas, pelo cálculo do alfa de Cronbach.

Após o estudo das propriedades psicométricas das escalas atitudinais, a hipótese $\mathrm{H}_{1}$ foi testada pela análise fatorial, realizada para a validade da medida. Para o teste das hipóteses $\mathrm{H}_{2}{ }^{\prime} \mathrm{H}_{3}$ e $\mathrm{H}_{4}$ a princípio empregou-se a modelagem de equações estruturais para investigar a possibilidade da ocorrência de um fator de segunda ordem, que agrupasse as medidas AFET, CC:LoAlt e CC:HiSac em uma única medida. Constatando-se a presença de um Heywood Case, fenômeno advindo de múltiplas causas possíveis, em que a variância de erro é negativa e as correlações superiores a 1, inviabilizou-se a interpretação dessa primeira análise fatorial confirmatória de segunda ordem. Assim, optou-se por realizar uma análise fatorial exploratória considerando as médias de cada uma das três dimensões.

Como forma de verificar o poder preditivo de cada uma das variáveis atitudinais sobre as intenções comportamentais de comprometimento organizacional, testou-se por regressão múltipla o efeito da interação de tais variáveis, sobre a EICCO. Sendo assim, 
a EICCO foi escolhida como critério para o estudo da validade concorrente das medidas atitudinais. Tendo por base tal tipo de validade, foram desenvolvidos dois modelos estruturais para verificar o inter-relacionamento das variáveis atitudinais, assim como o relacionamento entre tais variáveis e a EICCO. O primeiro modelo estrutural considerou a AFET, a CC:LoAlt e a CC:HiSac como variáveis preditoras da EICCO. Já o segundo modelo foi testado sem as variáveis CC:LoAlt e a CC:HiSac, considerando somente a influência da AFET sobre a EICCO. Para a composição dos modelos estruturais, utilizou-se como estimador o Maximum Likelihood (ML), técnica robusta, até mesmo quando há moderadas violações do pressuposto de normalidade (Anderson \& Gerbing, 1984).

\section{Resultados e Discussão}

\section{Estudo das propriedades psicométricas das medidas atitudinais}

Sendo empregados os métodos de análise fatorial pela TCT e pela TRI, a princípio, para a escala de comprometimento afetivo, verificou-se que todos os itens apresentaram nível de saturação item-total superior a 0,30. Na análise de itens não houve valores inesperados para o infit nem para o outfit. Para o estudo da consistência interna da Escala Afetiva, o valor obtido para o alfa de Cronbach foi de 0,86, não existindo itens que, caso fossem excluídos, melhorariam o nível geral de confiabilidade da medida.

Diferentemente da AFET, a escala instrumental não apresentou um padrão unidimensional. Ao serem calculados os eigenvalues, verificou-se que, para dois fatores, a variância explicada do construto foi de 56,12\%. A partir do terceiro fator, o valor do eigenvalue foi reduzido para 0,77, o que aponta uma solução bidimensional. Sendo utilizada a Análise de Componentes Principais para a extração dos fatores, com rotação oblíqua do tipo Promax, verificou-se que todos os valores de carga fatorial da escala de comprometimento instrumental foram superiores a 0,30. Entretanto, embora o item “Eu tenho tempo demais investido nesta organização para mudar" tenha saturado no fator "falta de alternativas", ele foi desenvolvido para compor o fator "sacrifícios percebidos". Sendo assim, por ir de encontro ao pressuposto teórico no qual se fundamenta este trabalho, tal item foi excluído das análises futuras. Não havendo também itens com valores insatisfatórios na análise de itens, verificou-se que o valor do alfa de Cronbach para CC:LoAlt foi de 0,66, enquanto para a CC:HiSac, foi de 0,70 .

Tendo por base o estudo da estrutura fatorial da escala instrumental, a hipótese $\mathrm{H}_{1}$ foi confirmada, dado que tal medida não se mostrou unidimensional, sendo dividida nas duas subdimensões previstas (CC:LoAlt e CC:HiSac). Sendo assim, procedeu-se ao teste das três hipóteses restantes.

\section{Testando a dimensionalidade do comprometimento organizacional}

Para o teste da hipótese $\mathrm{H}_{2}$, realizou-se a extração dos componentes principais das médias de cada dimensão, novamente com rotação Promax. Verificou-se a presença de dois fatores distintos. $O$ primeiro fator mostrou nitidamente compor a dimensão afetiva, com carga fatorial negativa da variável CC:LoAlt. Com o segundo fator ocorreu justamente o padrão inverso, com clara saturação da subdimensão CC:LoAlt e carga fatorial negativa da AFET. Já a subdimensão CC:HiSac mostrou saturação em ambos os fatores, como já era esperado, pois os sacrifícios percebidos para deixar a organização podem ser tanto de ordem emocional quanto de ordem instrumental. A variância explicada para dois fatores foi de 81,825\%. A Tabela 1 apresenta os valores de carga fatorial e a disposição dos fatores para as três variáveis atitudinais.

A partir dos resultados da análise fatorial exploratória entre as médias dos itens para cada variável atitudinal, pode-se constatar que o comprometimento organizacional atitudinal não forma uma estrutura coesa. Entretanto, tais achados não permitem ainda responder

Tabela 1. Análise fatorial das variáveis médias de comprometimento atitudinal.

\begin{tabular}{lccc}
\hline Variáveis (médias) & Fator 1 & Fator 2 & h2 \\
\hline AFET & 0,971 & $-0,73$ & 0,914 \\
CC:LoAlt & $-0,209$ & 0,940 & 0,833 \\
CC:HiSac & 0,369 & 0,672 & 0,708 \\
\hline
\end{tabular}


ao teste da hipótese $\mathrm{H}_{2}$, já que a matriz fatorial aponta uma distinção entre as variáveis AFET e CC:LoAlt, mas, pelos elevados valores de carga fatorial nos dois fatores, não se tem a garantia de qual dos fatores melhor representa o comprometimento organizacional atitudinal. Além disso, a subdimensão CC:HiSac demonstra forte saturação com os dois fatores, o que poderia apontar uma ambiguidade teórica.

Tendo em vista que as atitudes são um componente cognitivo que precede as intenções de comportamentos, foi escolhida a EICCO como medida de intenções de comprometimento organizacional, para funcionar como variável-critério da predição da AFET, da CC:LoAlt e da CC:HiSac. Se todas as variáveis forem preditoras da EICCO, logo se pressupõe que o comprometimento atitudinal deveria ser composto tanto pela dimensão afetiva quanto pela instrumental. Todavia, como alguns estudos teóricos têm apontado que a dimensão instrumental não compõe a estrutura fatorial do comprometimento organizacional atitudinal, não se espera que as subdimensões CC:LoAlt e CC:HiSac predigam significativamente as intenções comportamentais de comprometimento.

Para testar o poder preditivo das variáveis atitudinais individualmente sobre a EICCO, utilizou-se a princípio o método de regressão linear forward. A AFET foi a primeira variável testada para verificar a capacidade de maximização da previsão das intenções de comportamento, medida pela EICCO. Constatando-se a significância da AFET para a entrada do modelo de regressão, obteve-se uma correlação de ordem zero de $r=0,48$, com uma variância explicada de 23\% $[F(1,1867)=569,045$, $p<0,001]$. Com esses resultados, pode-se constatar que a variável AFET apresenta um poder preditivo representativo sobre posturas comportamentais de comprometimento organizacional.

Em um segundo momento, testou-se a relação entre CC:LoAlt e a EICCO. Embora tal subdimensão apresente significância estatística para entrada no modelo de regressão, verificou-se baixa correlação com a EICCO, a um $r=0,12(p<0,01)$ e uma baixíssima variância explicada de 1,3\%. Vale destacar ainda a obtenção de elevado valor do resíduo em relação ao modelo de regressão estimado, e um valor do F para a análise de variância bastante inferior ao obtido na regressão entre a variável AFET e a EICCO $[F(1,1867)=25,735, p<0,001]$.
Finalmente, testou-se a predição da CC:HiSac sobre a EICCO, encontrando-se resultados ainda menos expressivos. Correlacionando-se a um $r=0,02(p=0,455)$, CC:HiSac compartilha somente $0,7 \%$ da variância com a $\operatorname{EICCO}[\mathrm{F}(1,1867)=13,423, p<0,001]$.

Pelos resultados das análises de regressão, podese verificar que somente a dimensão afetiva (AFET) possui poder preditivo significativo sobre a EICCO, o que corroboraria as hipóteses $\mathrm{H}_{1}, \mathrm{H}_{2}$ e $\mathrm{H}_{3}$. No entanto, para a confirmação desses resultados, buscou-se realizar duas modelagens de equações estruturais entre as variáveis AFET, CC:LoAlt, CC:HiSac e a EICCO.

O primeiro modelo estrutural (Modelo 1) foi desenvolvido com as três variáveis atitudinais prevendo a EICCO. De acordo com os resultados do Modelo 1, pode-se verificar um alto nível de correlação entre AFET e EICCO ( $\beta=0,54, p<0,001)$; correlação nula entre CC:HiSac e EICCO ( $\beta=0,00, p<0,001)$; e correlação negativa entre CC:LoAlt e $\operatorname{EICCO}(\beta=-0,20, p<0,001)$.

O qui-quadrado do Modelo 1, com as quatro variáveis, foi $\chi^{2}=3600,207(p<0,001)$, melhorando para $\chi^{2}=2580,421(p<0,001)$, caso seja considerada somente a dimensão afetiva como variável preditora das intenções comportamentais de comprometimento organizacional (Modelo 2). Tendo em vista o aperfeiçoamento deste último modelo, foram incluídos quatro parâmetros (covariâncias) entre os erros de mensuração de alguns itens. Essas modificações foram realizadas com base no cálculo do Lagrange Multiplier Test. A princípio, foi realizada uma associação entre os erros de dois itens que se referem à tomada de defesa da organização $(\mathrm{MI}=263,653)$. Todos os demais parâmetros foram adicionados entre os erros de itens da AFET, que guardavam grande similaridade de conteúdo. Assim, foram associados os erros dos itens: "Eu sinto os problemas desta organização como se fossem meus" e "Sinto os objetivos dessa organização como se fossem os meus" (MI = 168,535); "Conversando com amigos, eu sempre me refiro a esta organização como uma grande instituição para a qual é ótimo trabalhar" e "Eu me sinto orgulhoso dizendo às pessoas que sou parte desta organização" (MI = 112,204); e"Esta organização tem um imenso significado pessoal para mim"e "Sinto que existe uma forte ligação afetiva entre mim e esta organização" $(\mathrm{Ml}=84,838)$. Após as quatro modificações, o valor do qui-quadrado foi reduzido para $\chi^{2}=1939,616(p<0,001)$. 
O modelo estrutural final (Modelo 2) encontrase disposto na Figura 1.

Como a estatística $\chi^{2}$ é bastante sensível a grandes amostras, pois aumentam o poder estatístico, resul- tando em significância com tamanhos de efeitos pequenos (Henson, 2006), foram considerados alguns índices de ajuste adicionais para determinar se o modelo é aceitável, conforme se vê na Tabela 2. Pode-se verificar
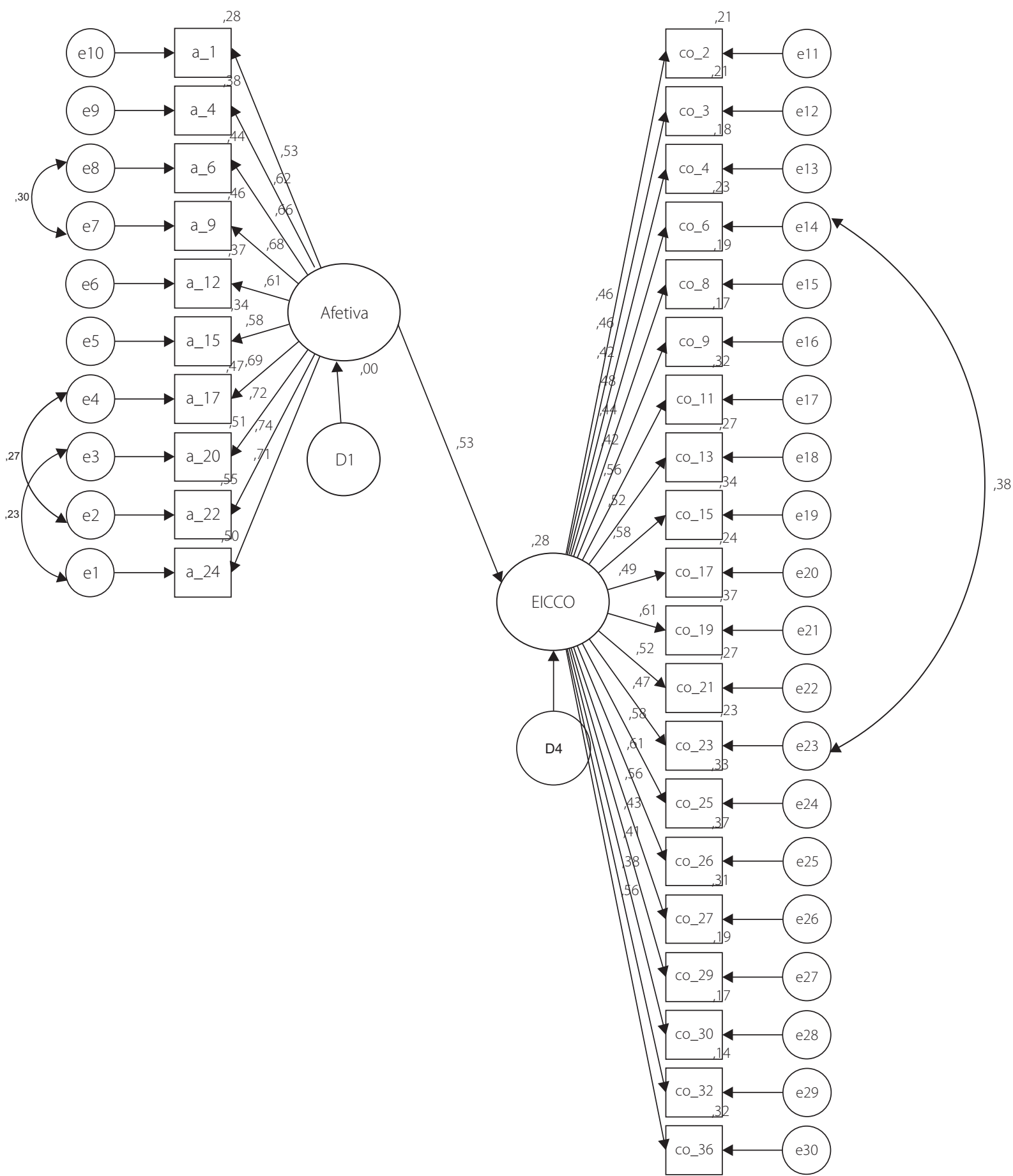

Figura 1. Modelo 2. Modelagem de equações estruturais com a variável afetiva predizendo a medida de intenções comportamentais.

470 Nota: EICCO: escala de intenções comportamentais de comprometimento organizacional. 
que somente o modelo com a variável AFET predizendo a EICCO apresenta um ajuste satisfatório na maior parte dos índices.

Todos os índices de ajuste desse segundo modelo foram superiores aos parâmetros esperados (Tabela 2).

Considerando assim os resultados dos modelos estruturais testados e das análises de regressão, não se pode aceitar que qualquer uma das subdimensões da base instrumental sirva como uma forte variável preditora direta da EICCO. Levando-se em conta somente as variáveis AFET, CC:HiSac e a EICCO em um modelo estrutural, verificou-se que a subdimensão CC:HiSac apresenta somente um efeito indireto moderado sobre $\operatorname{aEICCO}(\beta=0,19, p<0,001)$, derivado da associação com a AFET. Assim, embora os sacrifícios percebidos ao deixar a organização estejam associados com o sentimento de identificação, não se pode admitir que esses sacrifícios sejam uma base ou razão para a manutenção do vínculo entre trabalhador e organização, sendo uma variável à parte desse modelo estrutural. Esse achado pode ser corroborado pela variância explicada da AFET sobre a EICCO, que perfez um total de $28 \%$ e não se alterou com o acréscimo da CC:HiSac como variável preditora da EICCO.

À luz desse conjunto de resultados, pode-se admitir que as hipóteses $\mathrm{H}_{2} \mathrm{H}_{3}$ e $\mathrm{H}_{4}$ foram confirmadas, visto que as subdimensões da dimensão instrumental (CC:LoAlt e CC:HiSac) não funcionaram como boas preditoras para os itens de intenções comportamentais de comprometimento organizacional. Somente a dimensão afetiva demonstrou bom poder preditivo sobre a EICCO, possuindo uma fraca correlação com CC:LoAlt e uma correlação moderada com CC:HiSac, que tam-bém não apresentou efeitos diretos sobre a EICCO. Esse achado encontra suporte no trabalho de Salancik (1977), que afirma que a dimensão afetiva do comprometimento permite destacar a importância de características do trabalho que ampliem, no trabalhador, o"sentir-se responsável" por isso. O sentimento de responsabilidade induziria a um envolvimento comportamental com o trabalho que, por sua vez, geraria atitudes consistentes. Em conformidade, finalmente, com Mowday et al. (1982) e Solinger et al. (2008), pode-se admitir que o comprometimento organizacional atitudinal é um construto unidimensional, formado unicamente pela dimensão afetiva.

\section{Considerações Finais}

A maior parte dos estudos sobre comprometimento organizacional busca investigar quais as atitudes que melhor representam o construto. No entanto, decorridos praticamente 50 anos de pesquisas sobre o tema, ainda não há grande precisão sobre qual é a estrutura dimensional do comprometimento. Um dos fatores de indefinição sobre a constituição do comprometimento reside na dependência que a teoria possui de seus instrumentos de avaliação, assemelhando-se à falta de clareza conceitual dos testes de inteligência de Binet, traduzida na sua célebre frase: "Eu nomeio inteligência aquilo que medem os meus testes". Ao ser entendido como uma atitude, o comprometimento pode estar associado a inúmeras características psicológicas, como tipos de crenças, sentimentos e conações, sendo as medidas desenvolvidas sobre o construto tão somente

Tabela 2. Índices de ajuste estimados para as variáveis do comprometimento organizacional atitudinal.

\begin{tabular}{lccc}
\hline Nome & $\begin{array}{c}\text { Resultado (Modelo 1) } \\
\text { (AFET+ CC:LoAlt + CC:HiSac) > ElCCO }\end{array}$ & $\begin{array}{c}\text { Resultado (Modelo 2) } \\
\text { AFET > ElCCO }\end{array}$ & Parâmetro \\
\hline Normed Fit Índex (NFI) & 0,824 & 0,887 & \\
Incremental Fit Índex (IFI) & 0,848 & 0,908 & Acima de 0,90 \\
Comparative Fit Index (CFI) & 0,848 & 0,908 & \\
Goodness-of-Fit Index (GFI) & 0,895 & 0,932 & Acima de 0,90 \\
Adjusted Goodness-of-Fit Index (AGFI) & 0,882 & 0,921 & Valores pequenos \\
Root Mean square Residual (RMR) & 0,223 & 0,134 & Abaixo de 0,08 \\
Root Mean Square Error of Approximation (RMSEA) & 0,052 & 0,045 & \\
\hline
\end{tabular}

AFET: comprometimento atitudinal afetivo; CC:LoAlt: sub-dimensão falta de alternativas ou oportunidades de trabalho; CC:HiSac: sub-dimensão sacrifícios percebidos ao deixar a organização; EICCO: escala de intenções comportamentais de cidadania organizacional. 
uma parte que se acredita ser a mais relevante para a investigação.

Para o presente estudo, as medidas atitudinais empregadas buscaram investigar o comprometimento a partir de fatores - como identificação, interesses, envolvimento, preferências, internalização de valores - e da intenção de permanência e continuidade na organização. Um dos achados importantes deste estudo foi a constatação de que a visão de que o comprometimento estaria relacionado com questões instrumentais, como a necessidade de permanecer na organização, não faz parte da dimensionalidade do construto.

A falta de clareza sobre a dimensão instrumental pode ser atribuída a três fatores. Primeiro, à inexistência de uma estrutura fatorial coesa entre os itens dessa dimensão, constituída pelas subdimensões "sacrifícios percebidos" e "falta de oportunidades". Segundo, à indefinição de qual perspectiva teórica (atitudinal ou comportamental) melhor sustentaria seus pressupostos. Ao ser caracterizada como uma forma de side-bets, a dimensão instrumental pode acabar aproximando-se mais do enfoque comportamental do que o atitudinal, já que, como o próprio Becker (1990, p.33) preconiza, o comprometimento diz respeito ao "engajamento em linhas consistentes de ação" e envolve a rejeição de outras alternativas, como um elemento central do construto. Embora essas noções se coadunem com a concepção teórica da base instrumental, elas a entendem ora como uma atitude, ora como uma intenção de comportamento.

Por fim, o terceiro fator que torna imprecisa a dimensão instrumental como base do comprometimento organizacional relaciona-se com a ideia de permanência na organização: um trabalhador comprometido de forma instrumental seria aquele que deseja permanecer, ou pela percepção dos custos associados à sua saída, ou pela rejeição às demais alternativas de trabalho. Entretanto, atualmente, tem-se constatado que o desejo de permanecer na organização não faz parte do conceito de comprometimento, sendo um construto à parte (Menezes, 2006; Klein, Molloy \& Cooper, 2009). Como descreve Jaros (2009), trinta anos atrás, quando muitas pessoas esperavam construir carreira dentro de uma única organização, a noção ligada ao "desejo de permanência" era um consequente muito mais notável do

472 comprometimento. Contudo, atualmente, tanto empre- gador quanto empregado têm a consciência de que os contratos de trabalho podem ser de curto prazo e a permanência organizacional muito mais efêmera.

Diante dessas questões, se as medidas atitudinais de comprometimento organizacional negligenciam a modificação do próprio construto ao longo do tempo, assim como a não inclusão de termos comportamentais e/ou intenções de comportamento (Brown, 1996), as escalas que um dia foram válidas para avaliar o comprometimento organizacional, com o passar do tempo podem acabar por descrever o construto com um nível de precisão cada vez menor. A dimensão instrumental, ao carecer de falta de definição teórica e ao depender da ideia de permanência - que parece não ser um dos consequentes mais fortes para o comprometimento, nem tampouco integrar-se à definição do construto -, atualmente não aparenta integrar-se ao comprometimento organizacional como um dia parece tê-lo caracterizado.

Descartada a base instrumental como uma dimensão do comprometimento organizacional, o presente trabalho aponta que a base do vínculo estabelecido entre trabalhador e organização é formada tipicamente por um conjunto de fortes sentimentos e atitudes positivas dirigidas à organização, ou seja, pela dimensão afetiva. Destarte, o comprometimento organizacional, entendido a partir da perspectiva atitudinal, está intrinsecamente ligado a essa noção de afetividade, que, por sua vez, está associada ainda à ideia de lealdade e ao desejo de contribuir e dar energia para a organização (Mowday et al., 1982). Não obstante tais achados, faz-se importante que mais pesquisas sejam conduzidas, com outras medidas atitudinais e comportamentais, em diferentes contextos culturais, a fim de testar a hipótese de que o comprometimento organizacional atitudinal é um construto unidimensional.

\section{Referências}

Allen, N. J., \& Meyer, J. P. (1990). The measurement and antecedents of affective, continuance, and normative commitments to the organization. Journal of Occupational Psychology, 1 (63), 1-8.

Allen, N. J., \& Meyer, J. P. (1996). Affective, continuance and normative commitment to the organization: an examination of construct validity. Journal of Vocational Behaviour, 49 (3), 252-76. 
Anderson, J. C., \& Gerbing, D. W. (1984). The effect of sampling error on convergence, improper solutions, and goodness-of-fit indices for maximum likelihood confirmatory factor analysis. Psychometrika, 49 (2), 155-173.

Arnold, H. J., \& Feldman, D. C. (1982). A multivariate analysis of the determinants of job turnover journal of applied psychology. Journal of Applied Psychology, 67 (3), 350-360.

Bar-Hayim, A., \& Berman, G. S. (1992). The dimensions of organizational commitment. Journal of Organizational Behavior, 13 (4), 379-387.

Bastos, A. V. B. (1994). Comprometimento no trabalho: a estrutura dos vínculos do trabalhador com a organização, a carreira e o sindicato. Tese de doutorado não-publicada, Escola de Administração, Universidade de Brasília.

Becker, H. S. (1960). Notes on the concept of commitment. American Journal of Sociology, 66 (1), 32-42.

Bond, T. G., \& Fox, C. M. (2007). Applying the Rasch model: fundamentalmeasurement in the human sciences. Mahwah, NJ: Erlbaum.

Borges-Andrade, J. E., Afanasieff, R. S., \& Silva, M. S. (1989). Mensuração do comprometimento organizacional em instituições públicas. Anais da XIX Reunião Anual de Psicologia, Ribeirão Preto, SP.

Brown, R. B. (1996). Organizational commitment: clarifying the concept and simplifying the existing construct typology. Journal of Vocational Behavior, 49 (3), 230-251.

Carson, K., Carson, P., \& Bedeian, A. (1995). Development and construct validation of a career entrenchment measure. Journal of Occupational and Organizational Psychology, 68 (4), 301-320.

Cohen, A. (2003). Multiple commitments in the workplace: an integrative approach. Mahwah, NJ: Lawrence Erlbaum Associates.

Delobbe, N., \& Vandenberghe, C. (2000). A four-dimensional model of organizational commitment among Belgian employees. European Journal of Psychological Assessment, 16 (2), 125-138.

Hackett, R. D., Bycio, P., \& Hausdorf, P. A. (1994). Further assessments of Meyer and Allen's three-component model of organizational commitment. Journal of Applied Psychology, 79 (1), 15-23.

Hartmann, L. C., \& Bambacas, M. (2000) Organizational commitment: a multi method scale analysis and test of effects. International Journal of Organizational Analysis, 8 (1), 89-108

Henson, R. K. (2006). Effect-size measures and meta-analytic thinking in counseling psychology research. The Counseling Psychologist, 34 (5), 601-629.

Jaros, S. (2009). Measurement of commitment. In: H. J. Klein, T. E. Becker \& J. P. Meyer (Eds.), Commitment in organizations: accumulated wisdom and new directions (pp.347-382). Ohio: Routledge Academic.

Kelman, H. C. (1958). Compliance, identification, and internalization: three processes of attitude change? Journal of Conflict Resolution, 2(1), 51-60.
Klein, H. J., Molloy, J. C., \& Cooper, J. T. (2009). Conceptual foundations: construct definitions and theoretical representations of workplace commitments. In: H. J. Klein, T. E. Becker \& J. P. Meyer (Eds.), Commitment in organizations: accumulated wisdom and new directions (pp.3-36). Ohio: Routledge Academic.

Ko, J. W., Price, J. L., \& Mueller, C. W. (1997). Assessment of Meyer and Allen's three-component model of organizational commitment in South Korea. Journal of Applied Psychology, 82 (6), 961-973.

Labatmediene, L., Endriulaitiene, A., \& Gustainiene, L. (2007). Individual correlates of organizational commitment and intention to leave the organization. Baltic Journal of Management, 2 (2), 196-212.

Lee, J. A., \& Chulguen, Y. (2005). Factor structure of organizational commitment: differences between U.S. and South Korean samples. Psychological Reports, 96 (3), 595-602.

Mathieu, J. E., \& Zajac, D. M. (1990). A review and metaanalysis of the antecedents, correlates, and consequences of organizational commitment. Psychological Bulletin, 108 (2), 171-194

Mcgee, G. W., \& Ford, R. C. (1987). Two (or more?) dimensions of organizational commitment: reexamination of the affective and commitment scales. Journal of Applied Psychology, 72 (4), 638-642.

Medeiros, C. A. F. (2003). Comprometimento organizacional: um estudo de suas relações com características organizacionais e desempenho nas empresas hoteleiras. Tese de doutorado não-publicado, Faculdade de Economia, Administração e Contabilidade, Universidade de São Paulo.

Medeiros, C. A. F., Albuquerque, L. G., Marques, G. M., \& Siqueira, M. (2005). Um estudo exploratório dos múltiplos componentes do comprometimento organizacional. Revista Eletrônica de Administração, 11 (1), 1-16.

Medeiros, C. A. F., \& Enders, W. T. (1998). Validação do modelo de conceitualização de três componentes do comprometimento organizacional (Meyer e Allen, 1991). Revista de Administração Científica, 2 (3), 67-87.

Menezes, I. G. (2006). Escalas de Intenções Comportamentais de Comprometimento Organizacional (EICCO): concepção, desenvolvimento, validação e padronização. Dissertação de mestrado não-publicada, Programa de Pós-Graduação em Psicologia, Universidade Federal da Bahia, Salvador.

Meyer, J. P., \& Allen, N. J. (1984). Testing the side-bets theory of organizational commitment: some methodological considerations. Journal of Applied Psychology, 69 (3), 372-378.

Meyer, J. P., \& Allen, N. J. (1991). A three-component conceptualization of organizational commitment. Human Resource Management Review, 1 (1), 61-89.

Meyer, J. P., Allen, N. J., \& Gellatly, I. R. (1990). Affective and continuance commitment to the organization: evaluation of measures and analysis of concurrent and time-lagged relations. Journal of Applied Psychology, 75 (6), 710-720. 
Meyer, J. P., Allen, N. J., \& Smith, C. A. (1993). Commitment to organizations and occupations: extension and test of a three-component conceptualization. Journal of Applied Psychology, 78 (4), 538-551.

Meyer, J. P., Stanley, D. J., Herscovitch, L., \& Topolnytsky, L. (2002). Affective, continuance, and normative commitment to the organization: a meta-analysis of antecedents, correlates, and consequences. Journal of Vocational Behavior, 61 (1), 20-52.

Mowday, R. T., Porter, L. W., \& Steers, R. M. (1982). Employeeorganization linkages: the psychology of commitment, absenteeism, and turnover. New York: Academic Press.

Mowday, R. T., Steers, R. M., \& Porter, L. W. (1979). The measurement of organizational commitment. Journal of Vocational Behavior, $14(1)$, 43-77.

Powell, D. M., \& Meyer, J. P. (2004). Side-bet theory and the three-component model of organizational commitment. Journal of Vocational Behavior, 65, 157-177.

Randall, M. L., Cropanzano, R., Bormann, C. A., \& Biriulin, A. (1997). Organizational politics and organizational support as predictors of work attitudes, job performance, and organizational citizenship behavior. Journal of Organizational Behavior, 20 (2), 159-174.
Rego, A. (2003). Comprometimento organizacional e ausência psicológica: afinal, quantas dimensões? Revista de Administração de Empresas, 43 (4), 25-35.

Salancik, G. R. (1977). Commitment and the control of organizational behavior and belief. In B. M. Shaw \& G. R. Salancik (Orgs.), New directions in organizational behavior (pp.1-54). Chicago: St. Clair Press.

Shore, L. M., Tetrick, L. E., Shore, T. H., \& Barksdale, K. (2000). Construct validity of measures of Becker's side bet theory. Journal of Vocational Behavior, 57 (3), 428-444.

Solinger, O. N., van Olfen, W., \& Roe, R. A. (2008). Beyond the three-component model of organizational commitment. Journal of Applied Psychology, 93 (1), 70-83.

Troyanskaya, O., Cantor, M., Sherlock, G., Brown, P. O., Hastie, T., Tibshirani, etal. (2001). Missing value estimation methods for DNA microarrays. Bioinformatics, 17 (6), 520-525.

Wagner, M. B., Motta, V. T., \& Dornelles, C. (2004). SPSS passo a passo: statistical package for social sciences. Caxias do Sul: EDUCS.

Recebido em: 9/12/2009

Versão final reapresentada em: 26/4/2011

Aprovado em: 11/5/2011 